\title{
AdS space compactification and holographic mapping in the AdS/CFT correspondence
}

\author{
Henrique Boschi-Filho ${ }^{\text {a* }}$ and Nelson R. F. Braga ${ }^{\text {a }}$ \\ ${ }^{a}$ Instituto de Física, Universidade Federal do Rio de Janeiro, Caixa Postal 68528, 21941-972, Rio de \\ Janeiro, RJ, Brazil
}

Physical consistency of quantum fields in anti-de Sitter space time requires that the space must be compactified by the inclusion of a boundary where appropriate conditions are imposed. An interpretation for the presence of this boundary is found taking AdS as a limiting case of the space generated by a large number of coincident branes. The compactification of AdS leads to a discretization of the spectrum of bulk fields. As a consequence, we find a one to one mapping between the quantum states of scalar fields in AdS bulk and boundary. Using this mapping as an approximation for the dual relation between string dilaton field and scalar QCD glueballs the high energy QCD scaling is reproduced. We also use this map to estimate the ratio of scalar glueball masses.

\section{Introduction}

According to the Maldacena conjecture 1 the large $N$ limit of $S U(N)$ superconformal field theories in $n$ dimensions can be described by supergravity in $n+1$ dimensional anti de Sitter (AdS) spacetime times a compact manifold. This is known as the AdS/CFT correspondence. In this correspondence (see also refs. 23445] and for reviews see 678]) the AdS space shows up as a near horizon geometry of a set of coincident D3-branes. A Dirichlet $p$-brane or $\mathrm{D} p$-brane is a $p+1$ dimensional hyperplane where strings are allowed to end 910). The AdS/CFT correspondence can be understood as a realisation of the Holographic principle: "the degrees of freedom of a quantum theory with gravity can be mapped on the corresponding boundary" 111213 1415.

Precise prescriptions for the realization of the AdS/CFT correspondence were presented in 2 23 . by considering Poincare patches of AdS space. Originally AdS space was formulated using global coordinates. However Poincare coordinate system allows a simple definition for the flat boundary where the conformal field theory is defined as we will discuss in the next section. In section $\mathbf{3}$ we discuss the quantization of scalar bulk fields in AdS space which allow us to write a one to

\footnotetext{
*boschi@if.ufrj.br

†braga@if.ufrj.br
}

one mapping between bulk and boundary scalar fields. In section 4 we apply this holographic mapping to describe high energy QCD scaling of scalar glueballs. We also comment on the possibility of applying this holographic mapping to obtain the ratio of scalar glueball masses.

\section{AdS space and compactification}

AdS space can be described by global coordinates with finite ranges [16. In this context it was shown 17 18 that a consistent quantization can only be obtained if one supplements AdS space with a boundary in order to impose vanishing flux of particles and information there. Otherwise massless particles would be able to go to (or come from) spatial infinity in finite times and it would thus be impossible to define a Cauchy surface.

In the AdS/CFT correspondence one considers the AdS space represented by Poincaré coordinates $z, \vec{x}, t$ with metric

$d s^{2}=\frac{R^{2}}{(z)^{2}}\left(d z^{2}+(d \vec{x})^{2}-d t^{2}\right)$.

The boundary in this case is given by the surface $z=0$ plus a point defined by the limit $z \rightarrow \infty$. Thus a consistent quantization requires the inclusion of this surface and the extra point at infinity where the flux should be required to vanish. This 
is not possible using just one set of Poincaré coordinates. The point at infinity can not be accommodated in the original Poincare chart so that we have to introduce a second coordinate system $z^{\prime}, \vec{x}, t[19$. The point $z \rightarrow \infty$ can be represented in the second chart at some positive constant value $z^{\prime}=\delta$. The coordinates $z$ and $z^{\prime}$ of the two charts are related in this case by

$\frac{1}{z^{\prime}}=\frac{1}{\delta}-\frac{1}{z}$

with range $\delta \leq z^{\prime}<\infty$. The metric of the second coordinate system involves a Poincare like factor $\left(R / z^{\prime}\right)^{2}$ and can be written as 20$]$

$d s^{2}=\frac{R^{2}}{z^{\prime 2}}\left[\frac{\delta^{2} d z^{\prime 2}}{\left(z^{\prime}-\delta\right)^{2}}+\frac{\left(z^{\prime}-\delta\right)^{2}}{\delta^{2}}\left((d \vec{x})^{2}-d t^{2}\right)\right]$

Now the whole compact AdS space is described by the coordinate charts corresponding to eqs. (1) and (3). Further, with this second chart we find a horizon (infinite singularity in the spatial part of $\left.d s^{2}\right)$ at $z^{\prime}=\delta$. This was not apparent in the original Poincare chart. Note that there is another horizon at $z=0$. We will comment on this latter.

Let us now discuss the ten dimensional geometry generated by $N$ coincident D3-branes and its relation to the compactified AdS space. The D3brane metric can be written as 92

$$
\begin{aligned}
d s^{2} & =\left(1+\frac{R^{4}}{r^{4}}\right)^{-1 / 2}\left(-d t^{2}+d \vec{x}^{2}\right) \\
& +\left(1+\frac{R^{4}}{r^{4}}\right)^{1 / 2}\left(d r^{2}+r^{2} d \Omega_{5}^{2}\right)
\end{aligned}
$$

where we are using the same symbol $R$ for a constant that now satisfies $R^{4}=N / 2 \pi^{2} T_{3}$ where $T_{3}$ is the tension of a single D3-brane. The metric (4) has a horizon at $r=0$ with zero perpendicular area (apart from the $S_{5}$ term). It is interesting to look at the space corresponding to (4) in two limiting cases where it assumes simpler asymptotic forms: large and small $r$ compared to $R$. Considering first the region $r>>R$ (far from the horizon) the space is asymptotically a ten dimensional Minkowski space:

$\left(d s^{2}\right)_{f a r}=-d t^{2}+d \vec{x}^{2}+d r^{2}+r^{2} d \Omega_{5}^{2}$.
Now looking at the near horizon region $r<<R$ we can approximate the metric (4) as:

$d s^{2}=\frac{r^{2}}{R^{2}}\left(-d t^{2}+d \vec{x}^{2}\right)+\frac{R^{2}}{r^{2}} d r^{2}+R^{2} d \Omega_{5}^{2}$.

Changing the axial coordinate according to: $z=$ $R^{2} / r$, as in ref. [12], the metric that describes the brane system for $r / R<<1$ takes the form

$d s^{2}=\frac{R^{2}}{z^{2}}\left(d z^{2}+(d \vec{x})^{2}-d t^{2}\right)+R^{2} d \Omega_{5}^{2}$,

corresponding to $\mathrm{AdS}_{5} \times \mathrm{S}^{5}$. This corresponds to the Poincaré chart (11) apart from the $S_{5}$ factor. Note however that the horizon $r=0$ which corresponds to the limit $z \rightarrow \infty$ is not included in this chart as a consequence of the lack of a relation between $z$ and $r$ at $r=0$. It is interesting to note that from the point of view of a pure AdS space, one has to include this point as a requirement for a consistent quantization. Considering the brane space this point is already present, corresponding to the brane location. The inclusion of this point in the AdS space is possible by introducing one more coordinate chart as discussed before. So, indeed the horizon found in the second chart at $z^{\prime}=\delta$ corresponds to the brane horizon.

Let us now examine the large $r$ region of the D3-branes space. A massless particle moving in the $r \rightarrow \infty$ direction will arrive at an asymptotically Minkowski space as in eq.(5). Then it would spend an infinite time to reach spatial infinity. So, the Cauchy problem is well posed for the D3-branes space which is geodesically complete.

Further it is interesting to consider the limit $R \rightarrow \infty$ as suggested by the Maldacena conjecture 1. The larger we take $R$ the larger is the range of $r$ for which the AdS approximation (77) for the brane metric (4) holds. So one could naively disregard the asymptotic flat space region in this limit. Then one would find an AdS space without the boundary, where particles could enter or leave the space in finite times. This would lead to the absence of a well defined Cauchy problem.

If one chooses to disregard the flat space region, boundary conditions should be imposed at $r \rightarrow \infty$ in order to recover physical consistency. That means, in the limit $R \rightarrow \infty$ we should not 
represent the branes space by just a pure AdS space but rather by a compactified AdS including the hypersurface at $z=0$ besides the point $z$ at infinity.

It is interesting to note that if we consider the whole space to be of the AdS form (eq. (7)) there is a horizon with infinite area at $z=0$. This is not present in the D3-branes model and it is a consequence of closing the AdS space as required for physical consistency once the asymptotic flat space region has been removed.

\section{Quantum fields in AdS space and holo- graphic mapping}

Now we are going to consider scalar fields in a compact $n+1$ dimensional AdS space in Poincare coordinates. As discussed above we must introduce two coordinate charts and impose matching conditions on the fields in the region of their intersection. We take the first chart to be defined as $0 \leq z \leq z_{\max }$. We can take $z_{\max }$ arbitrarily large and map as much of AdS space as we want into this chart but the fact that $z_{\max }$ is finite implies the discretization of the spectrum associated with the coordinate $z$. In the region $0 \leq z \leq z_{\max }$ we can introduce the quantum fields 19

$$
\begin{aligned}
\Phi(z, \vec{x}, t) & =\sum_{p=1}^{\infty} \int \frac{d^{n-1} k}{(2 \pi)^{n-1}} \\
& \times \frac{z^{n / 2} J_{\nu}\left(u_{p} z\right)}{z_{\max } w_{p}(\vec{k}) J_{\nu+1}\left(u_{p} z_{\max }\right)} \\
& \times\left\{\mathbf{a}_{p}(\vec{k}) e^{-i w_{p}(\vec{k}) t+i \vec{k} \cdot \vec{x}}+\text { c.c. }\right\}
\end{aligned}
$$

where $w_{p}(\vec{k})=\sqrt{u_{p}^{2}+\vec{k}^{2}}$ and $u_{p}$ are such that $J_{\nu}\left(u_{p} z_{\max }\right)=0$. The operators $\mathbf{a}, \mathbf{a}^{\dagger}$ satisfy the commutation relations

$$
\begin{aligned}
& {\left[\mathbf{a}_{p}(\vec{k}), \mathbf{a}_{p^{\prime}}^{\dagger}\left(\vec{k}^{\prime}\right)\right]=2(2 \pi)^{n-1} w_{p}(\vec{k}) \delta_{p p^{\prime}} \delta^{n-1}\left(\vec{k}-\vec{k}^{\prime}\right)} \\
& {\left[\mathbf{a}_{p}(\vec{k}), \mathbf{a}_{p^{\prime}}\left(\vec{k}^{\prime}\right)\right]=\left[\mathbf{a}_{p}^{\dagger}(\vec{k}), \mathbf{a}_{p^{\prime}}^{\dagger}\left(\vec{k}^{\prime}\right)\right]=0}
\end{aligned}
$$

A consequence of the discretization of the field spectrum is that we can find a mapping between bulk and boundary states. Let us define a scalar field on the $n$ dimensional AdS boundary $z=0$

$$
\begin{aligned}
\Theta(\vec{x}, t) & =\frac{1}{(2 \pi)^{n-1}} \int_{-\infty}^{\infty} \frac{d^{n-1} K}{2 w(\vec{K})} \\
& \times\left\{\mathbf{b}(\vec{K}) e^{-i w(\vec{K}) t+i \vec{K} \cdot \vec{x}}+\text { c.c. }\right\}
\end{aligned}
$$

where $w(\vec{K})=\sqrt{\vec{K}^{2}+\mu^{2}}$ and the creationannihilation operators satisfy the canonical algebra

$\left[\mathbf{b}(\vec{K}), \mathbf{b}^{\dagger}\left(\vec{K}^{\prime}\right)\right]=2(2 \pi)^{n-1} w(\vec{K}) \delta\left(\vec{K}-\vec{K}^{\prime}\right)$

Following 21], we introduce a sequence of energy scales $\mathcal{E}_{1}, \mathcal{E}_{2}, \ldots$ and split the spectrum of the operator $\Theta$. For $0 \leq K \leq \mathcal{E}_{1}$ we introduce the relations between bulk and boundary operators

$$
\begin{aligned}
K^{\frac{n-2}{2}} \mathbf{b}\left(K, \phi, \theta_{\ell}\right) & =k^{\frac{n-2}{2}} \mathbf{a}_{1}\left(k, \phi, \theta_{\ell}\right) \\
K^{\frac{n-2}{2}} \mathbf{b}^{\dagger}\left(K, \phi, \theta_{\ell}\right) & =k^{\frac{n-2}{2}} \mathbf{a}_{1}^{\dagger}\left(k, \phi, \theta_{\ell}\right)
\end{aligned}
$$

where the moduli of the momenta are mapped onto each other as $k=g_{1}(K, \mu)$. Requiring that the canonical commutation relations for $\mathbf{a}$ and $\mathbf{b}$ are consistent with the above mapping we find a relation between momenta

$$
\begin{aligned}
g_{1}(K, \mu) & =\frac{1}{2} \frac{u_{1}^{2} C_{1}(\mu)}{\left(K+\sqrt{K^{2}+\mu^{2}}\right)} \\
& -\frac{K+\sqrt{K^{2}+\mu^{2}}}{2 C_{1}(\mu)} .
\end{aligned}
$$

For the other intervals we find similar mappings 21. This way we find a one to one mapping between bulk and boundary Fock spaces.

It is interesting to note that if we choose a convenient value of $C_{1}(\mu)$ and consider $\mu \ll K \ll \mathcal{E}_{1}$ we can approximate relation (14) as

$k \approx \frac{\mathcal{E}_{1} \mu}{2 K}$.

\section{High energy QCD scaling and glueball masses}

Using the mapping described above with $n=4$ we can obtain the high energy scaling of QCD glueballs at fixed angles. This can be done by relating the scattering amplitudes of scalar QCD glueballs with those of dilaton fields in AdS bulk. 
The assymptotic scalar glueballs are approximated by free scalar fields on the AdS boundary.

We start with the $S$ matrix for fixed angle scattering in the bulk (dilaton states) with 2 particles in the initial state and $m$ particles in the final state

$$
\begin{aligned}
& S_{\text {Bulk }} \\
& \left.=\left\langle\vec{k}_{3}, u_{1} ; \ldots ; \vec{k}_{m+2}, u_{1} ; \text { out }\right| \vec{k}_{1}, u_{1} ; \vec{k}_{2}, u_{1} ; \text { in }\right\rangle \\
& =\left\langle 0\left|\mathbf{a}_{\text {out }}\left(\vec{k}_{3}\right) \ldots \mathbf{a}_{\text {out }}\left(\vec{k}_{m+2}\right) \mathbf{a}_{\text {in }}^{+}\left(\vec{k}_{1}\right) \mathbf{a}_{\text {in }}^{+}\left(\vec{k}_{2}\right)\right| 0\right\rangle .
\end{aligned}
$$

Assuming the energy regime to be $\mu \ll K \ll$ $1 / R \ll k \ll 1 / \sqrt{\alpha^{\prime}}=\mathcal{E}_{1}$, where the supergravity approximation for string theory holds and then using the mapping (1213) between creationannihilation operators and the approximate relation (15) we obtain

$$
\begin{aligned}
& S_{\text {Bulk }} \times\left(\frac{k}{K}\right)^{m+2} \\
& \sim\left\langle 0\left|\mathbf{b}_{\text {out }}\left(\vec{K}_{3}\right) \ldots \mathbf{b}_{\text {out }}\left(\vec{K}_{m+2}\right) \mathbf{b}_{\text {in }}^{+}\left(\vec{K}_{1}\right) \mathbf{b}_{\text {in }}^{+}\left(\vec{K}_{2}\right)\right| 0\right\rangle \\
& \sim\left\langle\vec{K}_{3} \ldots \vec{K}_{m+2}, \text { out } \mid \vec{K}_{1}, \vec{K}_{2}, i n\right\rangle K^{(m+2)(d-1)} .(17)
\end{aligned}
$$

where the field $\Theta$ on the boundary has scaling dimension $d$ and then their in and out states are $|\vec{K}\rangle \cong K^{1-d} \mathbf{b}^{+}(\vec{K})|0\rangle$. This implies a relation between bulk and boundary $\mathrm{S}$ matrix elements

$S_{\text {Bulk }} \sim S_{\text {Bound. }}\left(\frac{\sqrt{\alpha^{\prime}}}{\mu}\right)^{m+2} K^{(m+2)(1+d)}$.

Finally the corresponding scattering amplitudes $\mathcal{M}$ are related by

$$
\begin{aligned}
\mathcal{M}_{\text {Bound. }} & \sim \mathcal{M}_{\text {Bulk }} S_{\text {Bound. }}\left(S_{\text {Bulk }}\right)^{-1}\left(\frac{K}{k}\right)^{4} \\
& \sim \mathcal{M}_{\text {Bulk }} K^{8-(m+2)(d+1)}\left(\frac{\sqrt{\alpha^{\prime}}}{\mu}\right)^{2-m} .
\end{aligned}
$$

In the particular energy regime considered we find

$$
\mathcal{M}_{\text {Boundary }} \sim K^{4-\Delta} \sim s^{2-\Delta / 2},
$$

where $\Delta=(m+2) d$ is the total scaling dimension of the scattering particles associated with glueballs on the four dimensional boundary and $K \sim \sqrt{s}$. This result corresponds to the expected QCD scaling behavior 2324 and was first reproduced in the AdS/CFT context by Polchinski and Strassler 25.
Extending the idea of this mapping to the case where there is a series of scalars $\Theta_{i}$ on the boundary we found a simple estimate for the ratio of scalar glueball masses 26] that in four dimensions reads:

$$
\frac{\mu_{i}}{\mu_{1}}=\frac{\chi_{2, i}}{\chi_{2,1}}
$$

where $\mu_{1}$ is the mass of the state $0^{++}, \mu_{i}$ are the masses of its excitations and $\chi_{2, i}$ are the zeros of the Bessel function $J_{2}$. The results of this estimate are close to the ones found in [27] starting with an AdS-Schwarzschild black hole metric, following Witten's suggestion 28. These results are also in good agreement with lattice QCD calculations 2930 .

More recently we have discussed the fact that this approximation for the ratio of the masses of scalar glueballs does not depend on the details of the bulk/boundary mapping discussed above. Alternatively it can be found taking an AdS slice as an approximation for the dual space to a confining gauge theory and identifying the dilaton modes with the glueball masses 31. We hope that this discussion can be useful to describe other processes and states of QCD even in an approximated way.

\section{Acknowledgments}

The authors are partially supported by CNPq, FINEP, FAPERJ and CAPES - Brazilian research agencies.

\section{REFERENCES}

1. J. Maldacena, Adv. Theor. Math. Phys. 2 (1998) 231.

2. S. S. Gubser , I.R. Klebanov and A.M. Polyakov, Phys. Lett. B428 (1998) 105.

3. E. Witten, Adv. Theor. Math. Phys. 2 (1998) 253.

4. W. Mueck and K. S. Viswanathan, Phys. Rev. D58(1998)041901.

5. D. Z. Freedman, S. D. Mathur, A. Matusis and L. Rastelli, Nucl. Phys. B546 (1999) 96.

6. O. Aharony, S.S. Gubser, J. Maldacena, H. Ooguri and Y. Oz, Phys. Rept. 323 (2000) 183. 
7. J. L. Petersen, Int. J. Mod. Phys. A14 (1999) 3597.

8. I. R. Klebanov, "TASI Lectures: Introduction to the AdS/CFT correspondence", hep-th 0009139

9. G. Horowitz and A. Strominger, Nucl. Phys. B360 (1991) 197.

10. J. Polchinski, "TASI Lectures on D-branes" hep-th 9611050

11. G. 't Hooft, "Dimensional reduction in quantum gravity" in Salam Festschrifft, eds. A. Aly, J. Ellis and S. Randjbar-Daemi, World Scientific, Singapore, 1993, gr-qc/9310026

12. L. Susskind, J. Math. Phys. 36 (1995) 6377.

13. L. Susskind and E. Witten, "The holographic bound in anti-de Sitter space", SU-ITP-98-39, IASSNS-HEP-98-44, hep-th 9805114

14. For a covariant generalisation of the Holographic principle see: R. Bousso, Class. Quant. Grav. 17 (2000) 997; JHEP 9906 (1999) 028.

15. D. Bigatti and L. Susskind, "TASI Lectures on the Holographic principle", hep-th 0002044

16. S. W. Hawking and G. Ellis, The Large Scale Structure of Space-time, Cambridge University Press, London, 1973.

17. S. J. Avis, C. J. Isham and D. Storey, Phys. Rev. D18 (1978) 3565.

18. P. Breitenlohner and D. Z. Freedman, Phys. Lett. B115(1982) 197; Ann. Phys. 144 (1982) 249.

19. H. Boschi-Filho and N. R. F. Braga, Phys. Lett. B505 (2001)263; Nucl. Phys. B608 (2001) 319.

20. H. Boschi-Filho and N. R. F. Braga, Phys. Rev. D66 (2002) 025005

21. H. Boschi-Filho and N. R. F. Braga, Phys. Lett. B525 (2002) 164.

22. H. Boschi-Filho and N. R. F. Braga, Phys. Lett. B560 (2003) 232.

23. V. A. Matveev, R.M. Muradian and A. N. Tavkhelidze, Lett. Nuovo Cim. 7 (1973) 719.

24. S. J. Brodsky and G. R. Farrar, Phys. Rev. Lett 31 (1973) 1153; Phys. Rev. D11 (1975) 1309.

25. J. Polchinski and M. J. Strassler, Phys. Rev. Lett. 88 (2002) 031601.
26. H. Boschi-Filho and N. R. F. Braga, "QCD/String holographic mapping and glueball mass spectrum", hep-th/0209080

27. C. Csaki, H. Ooguri, Y. Oz and J. Terning, JHEP 9901 (1999) 017.

28. E. Witten, Adv.Theor.Math.Phys. 2 (1998) 505-532.

29. C. J. Morningstar and M. Peardon, Phys. Rev. D 56 (1997) 4043.

30. M.J. Teper, in Confinement, Duality and Non-Perturbative Aspects of $Q C D$, NATO Advanced Study Institute, Cambridge 1997, hep-lat 9711011.

31. H. Boschi-Filho and N. R. F. Braga, JHEP05 (2003) 009. 